\title{
CONVEX CURVES MOVING HOMOTHETICALLY BY NEGATIVE POWERS OF THEIR CURVATURE*
}

\author{
JOHN URBAS ${ }^{\dagger}$
}

\begin{abstract}
We study immersed locally convex curves in $\mathbf{R}^{2}$ which move homothetically under flow by negative powers of their curvature.

1. Introduction. In $[11,12]$ we classified all complete noncompact embedded convex hypersurfaces in $\mathbf{R}^{n+1}$ which move homothetically under flow by a positive or negative power of their Gauss curvature. Furthermore, we observed that the embeddedness hypothesis could be dropped in the case $n \geq 2$ because the hypersurfaces in question are automatically embedded by virtue of results of Sacksteder [9] and van Heijenoort [13]. Our main aim here is to investigate the special case of locally convex curves in $\mathbf{R}^{2}$ moving homothetically by a negative power of their curvature.
\end{abstract}

Let $\gamma_{t}, t \in[0, T)$, be a family of curves in $\mathbf{R}^{2}$ given by smooth immersions $X_{t}=X(\cdot, t): I \rightarrow \mathbf{R}^{2}$ where $I$ is some open interval in $\mathbf{R}$. The curves $\gamma_{t}$ are said to move under $\kappa^{-\alpha}$ flow for some $\alpha>0$ if

$$
\frac{\partial X}{\partial t}(s, t)=\frac{1}{\kappa^{\alpha}(s, t)} \nu(s, t) \text { for all }(s, t) \in I \times[0, T),
$$

where $\kappa(\cdot, t)$ is the curvature of $\gamma_{t}$ and $\nu(\cdot, t)$ is the unit normal vectorfield of $\gamma_{t}$. Any smooth solution of (1.1) must have $\kappa$ of one sign. We use the conventions that for locally convex curves $\kappa \geq 0$ and $\nu$ points out of the convex region defined by the curve in the case that it bounds a convex region.

We are interested in homothetic solutions of (1.1), which means that $X_{t}=\phi(t) X_{0}$ for some positive function $\phi$. By the usual separation of variables argument we see that $X_{0}$ must satisfy

$$
\kappa^{-\alpha}[X]=\lambda\langle X, \nu\rangle
$$

and

$$
\phi^{\prime}(t) \phi^{-\alpha}(t)=\lambda
$$

for some nonzero constant $\lambda$. Since $\phi(0)=1$, we have

$$
\phi^{1-\alpha}(t)=1+\lambda(1-\alpha) t \quad \text { if } \quad \alpha \neq 1
$$

and

$$
\phi(t)=e^{\lambda t} \quad \text { if } \quad \alpha=1 .
$$

There are no solutions of (1.2) in the case $\lambda=0$ which corresponds to the trivial homothety $\phi(t) \equiv 1$.

We say $\gamma$ (or $X$ ) is an expanding solution if $\lambda>0$ and a contracting solution if $\lambda<0$. If $\alpha \neq 1$, we can rescale the solution so that $\lambda= \pm 1$. If $\alpha=1$, both sides of (1.2) scale the same way, and the behaviour of solutions may be different for different

${ }^{*}$ Received November 11, 1998; accepted for publication January 23, 1999.

$\dagger$ Centre for Mathematics and its Applications, School of Mathematical Sciences, Australian National University, Canberra ACT 0200 Australia (urbas@maths.anu.edu.au). 
values of $\lambda$. Expanding solutions are quite different from contracting solutions, so the two cases will be considered separately.

Expanding Solutions. A result of Chow and Gulliver [3] states that for any closed compact embedded solution of (1.1) with any $\alpha>0$, the quantity $\sup _{\gamma_{t}}\langle X, \nu\rangle-$ $\inf _{\gamma_{t}}\langle X, \nu\rangle$ remains bounded by a positive constant depending only on the initial curve $\gamma_{0}$ for as long as the flow exists. In particular, this implies that the only closed, compact, embedded solutions of (1.2) are circles about the origin of radius $\lambda^{1 /(\alpha-1)}$ for $\alpha \neq 1$ and of any radius if $\alpha=1$ (in this case we must take $\lambda=1$ ). For $\alpha \in(0,1]$ this is also a consequence of results in [5,10]. In fact, from [5,10] it follows that for $\alpha \in(0,1]$ the only closed compact locally convex immersed solutions of (1.2) are multiple covers of circular solutions. However, this is not true for $\alpha>1$ as we shall prove below.

THEOREM 1. Let $\alpha>1$ and $\beta=1 / \alpha$. Then for any pair of relatively prime positive integers $m>n$ satisfying

$$
\frac{1}{\sqrt{1-\beta}}<\frac{m}{n}<\frac{1}{1-\beta}
$$

there is a nontrivial closed compact immersed solution $\gamma$ of (1.2) with $\lambda=1$. This solution has total curvature $2 m \pi$ and it closes in $n$ periods of its curvature function.

By nontrivial we mean that $\gamma$ is not a multiple cover of a circle. We do not know whether there is only one such solution for each pair $m, n$ (up to rotations about the origin), or whether there are also solutions for $m, n$ outside the range in (1.6). We conjecture that there are no other smooth closed solutions (although there are closed solutions with a singularity-see Theorem 2), and that the solution obtained in Theorem 1 for given $m, n$ is unique up to homothety and rotation. This would follow immediately (as in [1]) from the strict monotonicity of the period integral, as will be explained in Section 2, but we have not been able to prove this.

We shall see below that every convex solution of (1.2) with $\lambda=1$ determines a positive solution of the equation

$$
u^{\prime \prime}+u=u^{\beta}, \quad \beta=1 / \alpha
$$

and vice versa. All nonconstant positive entire solutions of (1.7) are periodic if $\beta \in$ $(0,1)$, and give rise to closed curves precisely when the period is a rational multiple of $2 \pi$. The curves in Theorem 1 correspond to solutions of (1.7) with the initial conditions $u(0)=a, u^{\prime}(0)=0$, where $a \in(0,1)$. We shall see that these solutions have maximum value less than $\beta_{1}=[2 /(\beta+1)]^{1 /(1-\beta)}>1$. Consequently (see (2.7) and (2.8) below), the corresponding curves lie in the open ball $B_{\beta_{1}}(0)$. Other solutions of (1.2) arise from solutions of (1.7) with the initial conditions $u(0)=a, u^{\prime}(0)=0$ where $a \geq \beta_{1}$. These are described in Theorem 2 .

We introduce the following terminology. A solution of (1.2) is said to be maximal if it cannot be smoothly extended to a larger solution. A maximal solution $\gamma$ is said to be infinite if it has infinite length in both directions, semi-infinite if it is of infinite length, has one endpoint and $\kappa \rightarrow \infty$ at the endpoint, or finite if it has finite length and $\kappa \rightarrow \infty$ at both endpoints.

Theorem 2. Let $\alpha>1$. Then for any $a \geq \beta_{1}$ there is a unique, modulo $O(2)$, maximal solution $\gamma$ of (1.2) with $\lambda=1$ such that $\max _{p \in \gamma}|p|=a . \gamma$ is a finite, generally nonembedded, curve of finite total curvature $k$. Furthermore, $k$ is a continuous strictly 
decreasing function of $a$ and

$$
\lim _{a \rightarrow \beta_{1}} k=\frac{2 \pi}{1-\beta}, \quad \lim _{a \rightarrow \infty} k=\pi,
$$

where $\beta=1 / \alpha$. If $a=\beta_{1}$, the endpoints of $\gamma$ lie at the origin, so $\gamma$ is a closed curve. If $a>\beta_{1}$, the endpoints of $\gamma$ lie on $\partial B_{r}(0)$ where $r^{2}=a^{2}-[2 /(\beta+1)] a^{\beta+1}>0$, and the tangent vector to $\gamma$ at each endpoint is radial, pointing towards the origin at one endpoint and away from the origin at the other.

In the case $a=\beta_{1}$ the solutions are closed curves generally having a cusp or corner singularity at the origin. For certain values of $\beta$ (precisely when $2 \pi /(1-\beta)$ is an even multiple of $\pi$ ) the two endpoints meet in a $C^{1}$ fashion at the origin, but $\kappa \rightarrow \infty$ at the origin in any case.

Next we describe the expanding solutions of (1.2) in the case $\alpha \in(0,1)$.

THEOREM 3. Let $\alpha \in(0,1)$. Then every maximal solution $\gamma$ of $(1.2)$ with $\lambda=1$ is one of the following types of curves.

(i) An infinite covering of the unit circle.

(ii) A finite, generally nonembedded, locally convex curve of finite total curvature $k>\pi$, both endpoints of which lie on $\partial B_{r}(0)$ for some $r \in\left(0, \alpha_{*}\right)$ where

$$
\alpha_{*}=\sqrt{(1-\alpha) /(1+\alpha)}<1 .
$$

The tangent vector to the curve points radially at each endpoint, towards the origin at one endpoint and away from the origin at the other. There is exactly one such solution, modulo $O(2)$, for each $r \in\left(0, \alpha_{*}\right)$, and the total curvature $k$ is a continuous strictly increasing function of $r$ satisfying

$$
\lim _{r \rightarrow 0} k=\pi, \quad \lim _{r \rightarrow \alpha_{*}} k=\infty .
$$

(iii) An infinite, properly immersed, locally convex curve of finite total curvature $k>0$. There is exactly one such solution, modulo $O(2)$, for each $k>0$.

(iv) A semi-infinite, properly embedded, locally convex curve of finite total curvature, with its endpoint on $\partial B_{r}(0)$ for some $r>\alpha_{*}$, and tangent vector at the endpoint pointing radially. There is exactly one such solution, modulo $O(2)$, for each $r \in$ $\left(\alpha_{*}, \infty\right)$, and the total curvature $k$ is a continuous strictly decreasing function of $r$ satisfying

$$
\lim _{r \rightarrow \alpha_{*}} k=\infty, \quad \lim _{r \rightarrow \infty} k=0 .
$$

(v) A semi-infinite, locally convex, embedded curve of infinite total curvature. The curve has its endpoint on $\partial B_{\alpha_{*}}(0)$ with tangent vector pointing radially there, and the other end spirals asymptotically towards the unit circle from the inside. There is exactly one such solution, modulo $O(2)$.

(vi) An infinite, locally convex, embedded curve of infinite total curvature. One end of the curve spirals asymptotically to the unit circle from the outside, and the other end goes to infinity with finite total curvature outside $B_{R}(0)$ for any $R>1$. There is exactly one such solution, modulo $O(2)$.

The curve in Theorem 3 (ii) is a closed curve with a cusp when $k$ is an odd multiple of $\pi$. Theorem 3 (iii) is a generalization in the one dimensional case of the 
main result of [12]. The solutions in (iii) with $k<\pi$ are the only complete noncompact embedded solutions of (1.2).

The case $\alpha=1$ is somewhat special, because we cannot rescale the curve to make $\lambda=1$ in (1.3). The solutions behave differently in each of the three cases $\lambda=1, \lambda>1$ and $\lambda \in(0,1)$.

THEOREM 4. Let $\alpha=1$ and let $\gamma$ be a maximal solution of (1.2).

(i) If $\lambda=1$, then $\gamma$ is either an infinite covering of the unit circle, or a semiinfinite, properly embedded, locally convex curve of infinite total curvature. This solution has its endpoint on $\partial B_{r}(0)$ for some $r>0$ and tangent vector pointing radially there, and it spirals infinitely many times around the origin without selfintersection as it goes to infinity. There is exactly one such solution, modulo $O(2)$, for each $r>0$.

(ii) If $\lambda>1$, then $\gamma$ is either a semi-infinite, locally convex curve similar to the one described in (i), or an infinite, properly immersed curve of infinite total curvature. Each end of the curve spirals infinitely many times around the origin as it goes to infinity. There is exactly one such curve, modulo $O(2)$, with $\operatorname{dist}(0, \gamma)=r$ for each $r>0$ and each $\lambda>1$.

(iii) If $0<\lambda<1$, then $\gamma^{\prime}$ is a finite, properly immersed, locally convex curve of finite total curvature $k=\pi / \sqrt{1-\lambda}$, with both endpoints lying on $\partial B_{r}(0)$ for some $r>0$. The tangent vector to the curve points radially at each endpoint, towards the origin at one endpoint and away from the origin at the other. There is exactly one such solution, modulo $O(2)$, for each $r>0$ and each $\lambda \in(0,1)$.

Contracting Solutions. These are much less diverse than their expanding counterparts. To describe them we introduce some terminology.

Let $C$ be a closed convex cone in $\mathbf{R}^{2}$ with vertex at the origin and vertex angle less than $\pi$. We say that $C$ is a supporting cone for a finite $C^{1}$ embedded convex curve $\gamma$ if $\gamma \subset C$, the endpoints of $\gamma$ lie on $\partial C-\{0\}$ and $\gamma$ is tangential to $\partial C$ at the endpoints.

THEOREM 5. Let $\alpha>0$.

(i) If $\gamma$ is a maximal contracting solution of (1.2), then $\gamma$ is a finite, embedded, convex curve supported by a cone $C$ as above. In particular, there is no complete contracting solution.

(ii) If $\alpha=1$, then for any cone $C$ as above there is a unique $\lambda<0$ and a unique (up to homothety) maximal solution $\gamma$ of (1.2) with supporting cone $C$.

(iii) If $\alpha>0, \alpha \neq 1$, then for any cone $C$ as above and any $\lambda<0$ there is a unique (up to homothety) maximal solution $\gamma$ of (1.2) with supporting cone $C$.

We shall prove the above theorems in Sections 2 and 3, except for the proofs of some technical lemmas which are given in Section 5. In Section 4 we make some remarks about convex curves which move homothetically under flow by positive powers of their curvature. Most of the results in this section. are known, but we include them for comparison with flow by negative powers of the curvature.

2. Expanding Solutions. In this section we shall prove Theorems 1 to 4 . We 
begin by deriving equation (1.7). We assume that $\gamma$ is a convex solution of

$$
\kappa=\langle X, \nu\rangle^{-\beta}
$$

for some $\beta>0$. Since $\kappa>0$, we may parametrize $\gamma$ by its inverse Gauss map. More precisely, if we suppose that $\gamma$ is parametrized by its arclength $s$ measured from some point of $\gamma$, then we may express the tangent and normal vectors to $\gamma$ as

$$
\begin{aligned}
\tau & =\frac{d X}{d s}=(\cos \theta(s), \sin \theta(s)) \\
\nu & =(-\sin \theta(s), \cos \theta(s))
\end{aligned}
$$

where $\theta$ is some smooth, strictly increasing function of $s$. The Frenet equations

$$
\frac{d^{2} X}{d s^{2}}=-\kappa \nu, \quad \frac{d \nu}{d s}=\kappa \frac{d X}{d s},
$$

together with (2.2), then imply that $\kappa=-d \theta / d s$. Note that our conventions regarding $\nu$ and $\kappa$ lead to different signs in (2.3) than is usual. Now let $u=u(\theta)=\langle X, \nu\rangle$. Then using the Frenet equations we derive

$$
u^{\prime \prime}+u=\kappa^{-1}
$$

and hence, by (2.1),

$$
u^{\prime \prime}+u=u^{\beta}
$$

Here and below ' denotes differentiation with respect to $\theta$. Since

$$
X=\langle X, \nu\rangle \nu+\langle X, \tau\rangle \tau=u \nu+u^{\prime} \tau,
$$

$\gamma$ is completely determined by $u$. The total curvature of $\gamma$ is simply the length of the interval on which $u$ is defined. To prove the results stated in the introduction, it is sufficient therefore to prove corresponding assertions about positive solutions of (2.5). Note that $\gamma$ is complete, noncompact and properly immersed if and only if

$$
|X|^{2}=|u(\theta)|^{2}+\left|u^{\prime}(\theta)\right|^{2} \rightarrow \infty
$$

as $\theta$ approaches the endpoints of the interval on which $u$ is defined. From (2.5) and (2.6) we see that

$$
\begin{aligned}
\frac{d}{d \theta}|X(\theta)|^{2} & =\frac{d}{d \theta}\left(|u(\theta)|^{2}+\left|u^{\prime}(\theta)\right|^{2}\right) \\
& =2 u u^{\prime}+2 u^{\prime} u^{\prime \prime} \\
& =2 u^{\beta} u^{\prime}
\end{aligned}
$$

so the curve $\gamma$ determined by $u$ has no self-intersections if either $u^{\prime}>0$ or $u^{\prime}<0$ everywhere. Furthermore, any portion of the curve corresponding to an interval on which $u^{\prime}>0$ or $u^{\prime}<0$ has no self-intersections.

We now study positive solutions of (2.5). By standard ODE theory the initial value problem

$$
u^{\prime \prime}+u=u^{\beta}, \quad u(0)=a, \quad u^{\prime}(0)=b
$$

has a unique solution for any $a>0$ and $b \in \mathbf{R}$, and any positive solution of (2.5) defined on some interval can be smoothly continued for as long as it remains positive and bounded. Thus we shall always assume without further comment that any solution 
is defined on its maximal interval of existence. The behaviour of solutions depends on the value of $\beta$, so we consider a number of cases separately.

Case 1. $0<\beta<1$. In this case we see that the function $g(t)=t^{\beta}-t$ is positive and strictly increasing for $0<t<1$, negative and strictly decreasing for $t>1$, and zero only at $t=0$ and $t=1$. Thus for any $a \in(0,1)$ the solution of

$$
u^{\prime \prime}+u=u^{\beta}, \quad u(0)=a, \quad u^{\prime}(0)=0,
$$

is strictly convex where $0<u<1$ and strictly concave where $u>1$. From the above properties of $g$ it is evident that $u$ has a local minimum at 0 (since $a \in(0,1)$ ) and that $u$ must achieve a local maximum greater than 1 at some finite $\theta>0$. Since (2.5) is invariant under the transformation $\theta \mapsto-\theta, u$ is an even function. Furthermore, since (2.5) is autonomous, $u$ must be symmetric about any local maximum or minimum. This implies that $u$ is periodic.

By (2.2), the tangent and normal vectors to the curve $\gamma$ determined by $u$ are $2 \pi$ periodic with respect to $\theta$, so it is clear that $\gamma$ is a closed curve precisely when the period of $u$ is a rational raultiple of $2 \pi$. We now derive an expression for the period $T$ of $u$. We have $u^{\prime} \geq 0$ for $0 \leq \theta \leq T / 2$ and $u$ has a minimum at 0 and a maximum at $T / 2$. Multiplying (2.5) by $u^{\prime}$ and integrating from 0 to $\theta \in(0, T / 2]$ we obtain, since $u(0)=a$ and $u^{\prime}(0)=0$,

$$
\left|u^{\prime}(\theta)\right|^{2}=F(a)-F(u)
$$

where

$$
F(s)=s^{2}-\frac{2}{\beta+1} s^{\beta+1} .
$$

Note that $F(s)=0$ at $s=0$ and at $s=\beta_{1}=[2 /(\beta+1)]^{1 /(1-\beta)}>1, F<0$ on $\left(0, \beta_{1}\right)$ and $F>0$ for $s>\beta_{1}$. Furthermore, $F$ attains its minimum value $\beta_{*}=$ $(\beta-1) /(\beta+1)<0$ at $s=1$. For each $a \in[0,1)$ there is a unique $b \in\left(1, \beta_{1}\right]$ such that $F(b)=F(a)$. It is clear that $b$ is a continuous function of $a$. Using the facts that $u^{\prime}>0$ in $(0, T / 2)$ and $u^{\prime}(0)=u^{\prime}(T / 2)=0$, we see from (2.11) that

$$
T=2 \int_{0}^{T / 2} \frac{u^{\prime}(\theta) d \theta}{\sqrt{F(a)-F(u(\theta))}}=2 \int_{a}^{b} \frac{d s}{\sqrt{F(a)-F(s)}} .
$$

To prove Theorem 1 it is sufficient to show that $T$ is continuous with respect to $a$ for $0<a<1$ and

$$
\lim _{a \rightarrow 0^{+}} T=\frac{2 \pi}{1-\beta}, \quad \lim _{a \rightarrow 1^{-}} T=\frac{2 \pi}{\sqrt{1-\beta}} .
$$

The continuity of $T$ follows from the fact that solutions of (2.10) depend continuously (in fact smoothly) on $a$ for $a \in(0,1)$, while $(2.14)$ is proved in Lemma 5.1. This completes the proof of Theorem 1.

Remark. We conjecture that $T$ is a strictly decreasing function of $a$, but we have not been able to prove this. As mentioned in the introduction, this would imply that the solutions obtained in Theorem 1 are unique up to homothety and rotation.

To prove Theorem 2, we first observe that the initial value problem (2.10) is solvable for any $a>0$. In addition, the energy of any solution

$$
E(u)=\left|u^{\prime}\right|^{2}+F(u)
$$


where $F$ is given by (2.12), is completely determined by the initial conditions $u(0)=a$ and $u^{\prime}(0)=b$. In particular, for $a \geq \beta_{1}$ and $b=0$ we see that $u \leq a$ and $u^{\prime} \leq 0$ on $[0, T)$, where $(-T, T)$ is the maximal interval on which $u$ remains positive, and in addition

$$
\left|u^{\prime}\right|^{2}+F(u)=F(a) \geq F\left(\beta_{1}\right)=0 .
$$

By considerations similar to those leading to (2.13) we see that

$$
T=\int_{0}^{a} \frac{d s}{\sqrt{F(a)-F(s)}} .
$$

If $a=\beta_{1}$, then $T\left(\beta_{1}\right)=\pi /(1-\beta)$ by Lemma 5.2 , and $u(T)=0, u^{\prime}(T)=0$, so $u$ extends to a nonnegative periodic function with period $2 \pi /(1-\beta)$. This does not contradict the uniqueness theorem for solutions of the initial value problem (2.10) because $\beta<1$. If $a>\beta_{1}$, then $u^{\prime}(T)=-\sqrt{F(a)}<0$, and by Lemma 5.2

$$
\lim _{a \rightarrow \beta_{1}^{+}} T=\frac{\pi}{1-\beta}, \quad \lim _{a \rightarrow \infty} T=\frac{\pi}{2} .
$$

These solutions give the curves described in Theorem 2; (1.8) follows directly from (2.17). This completes the proof of Theorem 2 .

Case 2. $\beta>1$. In this case there are several types of positive solutions of (2.5). It is convenient to classify these according to their energy levels. Note that $F$ has zeros at $s=0$ and at $s=\beta_{1}=[(\beta+1) / 2]^{1 /(\beta-1)}>1$, and now, since $\beta>1, F$ attains its maximum value $\beta_{*}=(\beta-1) /(\beta+1) \in(0,1)$ at $s=1$. Furthermore, $F$ is strictly increasing for $0<s<1$ and strictly decreasing for $s>1$.

Positive solutions of (2.5) can now be classified according to their energy levels as follows.

(i) $E(u)<\beta_{*}$. There are two subcases to consider. If $0 \leq E(u)<\beta_{*}$, then there are precisely two numbers $a_{1} \in[0,1)$ and $a_{2} \in\left(1, \beta_{1}\right]$ such that $F\left(a_{i}\right)=E(u)$ for $i=1,2$. Thus for the solutions of $(2.5)$ with $u(0)=a_{i}, i=1,2$, we must have $u^{\prime}(0)=0$. For $a_{1}=0$ we obtain the trivial solution $u \equiv 0$ which is of no interest, while for $a_{1} \in(0,1)$ we obtain a concave solution with maximum value $a_{1}<1$, defined on a bounded interval which is symmetric about the origin. These solutions give the curves described in Theorem 3 (ii). Let $(-T, T)$ be the interval of existence of the solution of $(2.5)$ with $u(0)=a \in(0,1)$ and $u^{\prime}(0)=0$. Then $u^{\prime} \leq 0$ on $[0, T)$ and

$$
-u^{\prime}(\theta)=\sqrt{F(a)-F(u(\theta))}
$$

so

$$
T=\int_{0}^{a} \frac{d s}{\sqrt{F(a)-F(s)}} .
$$

By Lemma 5.3, $T$ is a strictly increasing function of $a, T>\pi$ and

$$
\lim _{a \rightarrow 0^{+}} T=\pi, \quad \lim _{a \rightarrow 1^{-}} T=\infty .
$$

The fact that $T>\pi$ is also geometrically evident.

For $a_{2}>1$ we obtain a convex solution with minimum value $a_{2}>1$ at the origin. If $E(u)<0$, then there is precisely one number $a>\beta_{1}$ such that $F(a)=E(u)$, and the solution is also of this type. These solutions give the curves described in Theorem 3 
(iii). Let $(-T, T)$ be the interval of existence of the solution of (2.5) with $u(0)=a>1$ and $u^{\prime}(0)=0$. Then as above we see that

$$
T=\int_{a}^{\infty} \frac{d s}{\sqrt{F(a)-F(s)}}
$$

By Lemma 5.4, $T$ is strictly decreasing and

$$
\lim _{a \rightarrow 1^{+}} T=\infty, \quad \lim _{a \rightarrow \infty} T=0 .
$$

Since $T$ depends continuously on $a$ for $a \in(1, \infty)$, it follows that $T$ takes every value in $(0, \infty)$ exactly once. This implies the uniqueness assertion in Theorem 3 (iii).

(ii) $E(u)>\beta_{*}$. Since $F(s) \leq \beta_{*}$, for these solutions we have $\left|u^{\prime}\right|^{2} \geq E(u)-\beta_{*}>0$, so $u^{\prime}$ cannot change sign, and $u$ must take the value zero somewhere. Thus modulo a reflection and translation these solutions are obtained by solving (2.5) with the initial conditions $u(0)=0, u^{\prime}(0)=b>0$ where $b^{2}>\beta_{*}$. Note that the uniqueness theorem for the initial value problem is applicable at $u=0$ because $\beta>1$. These solutions give the curves described in Theorem 3 (iv). Since $u^{\prime}$ is bounded away from zero, $u$ takes every positive value exactly once, and $u(\theta) \rightarrow \infty$ as $\theta \rightarrow T$ for some finite $T$. In fact, we can get an explicit expression for $T$ by the argument used to derive (2.13), namely

$$
T=\int_{0}^{\infty} \frac{d s}{\sqrt{b^{2}-F(s)}} .
$$

Since $F(s) \leq \beta_{*}<b^{2}$ for all $s \geq 0$ and $b^{2}-F(s)=O\left(s^{\beta+1}\right)$ as $s \rightarrow \infty$, we see that $T$ is finite for any $b>\sqrt{\beta_{*}}$. Furthermore, the integrand in (2.22) (and hence also $T$ itself) is strictly decreasing with respect to $b$ for $b \in\left(\beta_{*}, \infty\right), T$ is continuous with respect to $b$, and by Lemma 5.5

$$
\lim _{b \rightarrow \sqrt{\beta_{*}}} T=\infty, \quad \lim _{b \rightarrow \infty} T=0 .
$$

(iii) $E(u)=\beta_{*}$. In this case the solution of $(2.5)$ with $u(0)=a, u^{\prime}(0)=b$, must satisfy

$$
\left|u^{\prime}\right|^{2}+F(u)=b^{2}+F(a)=\beta_{*} .
$$

Since the maximum value $\beta_{*}$ of $F$ is attained only at $s=1$, we must have $\left|u^{\prime}\right|^{2}>0$ unless $u(\theta)=1$ and $u^{\prime}(\theta)=0$ at some point $\theta$, in which case the uniqueness theorem for solutions of the initial value problem implies that $u \equiv 1$. Thus nonconstant solutions with energy equal to $\beta_{*}$ never take the value 1 . These solutions can therefore be characterized as follows: for each $b>\sqrt{\beta_{*}}$ there are precisely two numbers $a_{1} \in(0,1)$ and $a_{2} \in\left(1, \beta_{1}\right)$ such that $(2.24)$ holds with $a$ replaced by $a_{1}$ and $a_{2}$. For each such $b$ there are two nonconstant positive solutions of (2.5), namely the solutions with the initial conditions $u(0)=a_{i}, u^{\prime}(0)=b$ for $i=1,2$. Thus, possibly after a change of variable $\theta \mapsto-\theta$, for $i=1$ we obtain a concave strictly increasing solution $u_{1}$ with $0<u_{1}<1$ and which takes every value in $(0,1)$, and for $i=2$ we obtain a convex strictly increasing solution $u_{2}>1$ which takes every value in $(1, \infty)$.

The solution $u_{1}$ must be equal to zero somewhere, since it is strictly increasing and concave, so it is a translation of the solution of (2.5) with the initial condition $u(0)=0$, $u^{\prime}(0)=\sqrt{\beta_{*}}$, with interval of existence $(0, \infty)$. Thus it is unique up to translation and reflection. Since $|u(\theta)|^{2}+\left|u^{\prime}(\theta)\right|^{2} \rightarrow 1$ as $\theta \rightarrow \infty$, the curve $\gamma$ determined by $u$ 
starts at a point $p_{0}$ with $\left|p_{0}\right|=\sqrt{\beta_{*}}=\alpha_{*}$, spirals infinitely many times around the origin and asymptotically approaches the unit circle from the inside. This is the curve described in Theorem $3(\mathrm{v})$.

The solution $u_{2}$ is defined on an interval $(-\infty, T)$ for some finite $T$ and $u_{2}(\theta) \rightarrow 1$ as $\theta \rightarrow-\infty$. By the same considerations as before we see that

$$
T=\int_{a_{2}}^{\infty} \frac{d s}{\sqrt{\beta_{*}-F(s)}},
$$

so $T$ is clearly finite for any $a_{2}>1$ and

$$
\lim _{a_{2} \rightarrow 1^{+}} T=\infty
$$

by Lemma 5.6. We assert in addition that $u_{2}$ is unique up to translations. To prove this let $u, v$ be two solutions of (2.5) of this type. By translating $u$ and $v$ we can arrange that $u(0)=v(0)>1$. But then $u^{\prime}(0)=\sqrt{\beta_{*}-F(u(0))}=\sqrt{\beta_{*}-F(v(0))}=v^{\prime}(0)$, so $u \equiv v$ by the uniqueness theorem.

As above we see that the curve $\gamma$ determined by $u_{2}$ has no self-intersections. In one direction it goes to infinity with finite total curvature, and in the other it spirals infinitely many times around the origin and asymptotically approaches the unit circle from the outside. This is the curve described in Theorem 3 (vi). This completes the proof of Theorem 3.

Case 3. $\beta=1$. The proof of Theorem 4 is much simpler because we can solve equation (2.5) explicitly. Modulo translations and reflections the solutions are positive constants and linear functions if $\lambda=1$, positive multiples of either $\sinh \theta$ or $\cosh \theta$ if $\lambda>1$, and positive multiples of $\cos \theta$ if $0<\lambda<1$; in each case these are restricted to the intervals on which they are positive.

3. Contracting Solutions. In this section we prove Theorem 5 .

Let $\gamma$ be a maximal solution of (1.2) with $\lambda<0$, say $\lambda=-1$ for convenience. Since $\gamma$ is locally convex and smooth in its interior, $\kappa>0$ and $-\langle X, \nu\rangle>0$, which means that at least locally $\gamma$ lies on the opposite side from the origin of each of its tangent lines. But then it is clear that $\gamma$ cannot be a closed curve, nor can it contain any closed subcurve. Thus $\gamma$ must be embedded, and moreover, its total curvature must be less than $\pi$.

Since $\gamma$ is assumed to be smooth in its interior, $0 \notin$ interior $\gamma$ by (1.2). Thus if $\gamma$ is infinite, then $-\langle X, \nu\rangle \leq d=\operatorname{dist}(0, \gamma)$. Rearranging (1.2) and integrating we obtain

$$
\pi \geq \int_{\gamma} \kappa \geq \int_{\gamma} d^{-\beta}=\infty
$$

where $\beta=1 / \alpha$. So $\gamma$ cannot be infinite, and a very similar argument shows that it cannot be semi-infinite either. Thus $\gamma$ must be finite.

Now let $C$ be the intersection of all closed halfspaces $H$ of $\mathbf{R}^{2}$ with $0 \in \partial H$ and $\gamma \subset H$. Then $C$ is a closed convex cone with vertex at the origin, and $\gamma \subset C$. Furthermore, $\gamma$ meets $\partial C$ only at its endpoints. Since $\gamma$ is maximal, $\langle X, \nu\rangle=0$ at the endpoints, so $\gamma$ is tangential to $\partial C$ there. Finally, the vertex angle of $C$ is less than $\pi$ because it is equal to the total curvature of $\gamma$. This completes the proof of Theorem 5 (i). 
To prove Theorem 5 (ii) it is sufficient to show that for any $\mu \in(0, \pi / 2)$ there is a negative solution $u$ of

$$
\begin{aligned}
u^{\prime \prime}+u & =|\lambda|^{\beta}(-u)^{\beta} \text { in }(-\mu, \mu), \\
u & =0 \text { at } \theta= \pm \mu
\end{aligned}
$$

To solve this problem it is convenient to normalize so that $\lambda=-1$ if $\beta \neq 1$ and to replace $u$ by $-u$. Thus we need to prove the existence of a positive solution $v$ of

$$
\begin{aligned}
v^{\prime \prime}+v & =-v^{\beta} \quad \text { in } \quad(-\mu, \mu), \\
v & =0 \quad \text { at } \quad \theta= \pm \mu,
\end{aligned}
$$

if $\beta \neq 1$, and of

$$
\begin{aligned}
v^{\prime \prime}+v & =-|\lambda| v \quad \text { in } \quad(-\mu, \mu), \\
v & =0 \quad \text { at } \quad \theta= \pm \mu,
\end{aligned}
$$

if $\beta=1$. In the second case $v(\theta)=\cos (\pi \theta / 2 \mu)$ is the required solution, with $|\lambda|+1=$ $(\pi / 2 \mu)^{2}$. For any $\mu \in(0, \pi / 2)$ there is a unique $\lambda<0$ satisfying this equality, and $v$ is unique up to multiplication by positive constants.

If $\beta>0, \beta \neq 1$, the existence of positive solutions of (3.2) is no doubt well known. We do not know of a reference for this result, so we give a proof here.

We consider the initial value problem

$$
v^{\prime \prime}+v=-v^{\beta}, \quad v(0)=a>0, \quad v^{\prime}(0)=0 .
$$

This has a unique positive solution $v$ for each $a>0$, and $v$ is defined on some bounded interval $[-T, T]$ with $v( \pm T)=0$, since $v$ is even and strictly concave wherever it is positive. As before we see that

$$
T=\int_{0}^{a} \frac{d s}{\sqrt{F(a)-F(s)}} .
$$

By Lemma 5.7, $T$ is strictly increasing with

$$
\lim _{a \rightarrow 0^{+}} T=0, \quad \lim _{a \rightarrow \infty} T=\frac{\pi}{2} \quad \text { if } \quad 0<\beta<1,
$$

and strictly decreasing with

$$
\lim _{a \rightarrow 0^{+}} T=\frac{\pi}{2}, \quad \lim _{a \rightarrow \infty} T=0 \quad \text { if } \quad \beta>1 .
$$

Since $T$ depends continuously on $a$, there is a unique $a>0$ so that $T=\mu$. Since any positive solution of (3.2) is even, it follows from the above that (3.2) has a unique positive solution for any $\mu \in(0, \pi / 2)$.

4. Positive Powers. In this section we make some remarks about convex curves moving homothetically by positive powers of their curvature, i.e., solutions of

$$
\kappa^{\alpha}[X]=-\lambda\langle X, \nu\rangle \text {. }
$$

In this case solutions expand if $\lambda>0$ and contract if $\lambda<0$. There are many such results, particularly in the case $\alpha=1$. It is geometrically clear that there is no closed compact convex solution of (4.1) unless $\lambda<0$. In addition, circles about the origin of radius $\lambda^{-1 /(1+\alpha)}$ are solutions of (4.1). For $\alpha \in(0,1 / 8)$ there are other embedded 
compact convex solutions, and these have been classified by Andrews [2]. For $\alpha=1$ there is a family of nonembedded compact locally convex solutions of (4.1) first found by Mullins [8] and classified by Abresch and Langer [1]. Similar families of solutions can be shown to exist for other values of $\alpha$, but we do not know whether there is a complete description of these in the literature.

Contracting Solutions. Similarly to Section 2, all strictly convex solutions of (4.1) with $\lambda=-1$ are determined by positive solutions of the equation

$$
u^{\prime \prime}+u=u^{-\beta}, \quad \beta=1 / \alpha,
$$

and vice versa. If we now let

$$
F(s)=\left\{\begin{array}{l}
s^{2}-\frac{2}{1-\beta} s^{1-\beta} \quad \text { if } \beta \neq 1, \\
s^{2}-2 \log s \quad \text { if } \quad \beta=1,
\end{array}\right.
$$

then all positive solutions of (4.2) can be classified according to their energy $E(u)$ which is defined by

$$
E(u)=\left|u^{\prime}\right|^{2}+F(u) .
$$

Case 1. $\beta \geq 1$. In this case $F(s) \rightarrow \infty$ as $s \rightarrow 0$ or $s \rightarrow \infty, F$ is strictly convex and has its minimum value $\beta_{*}>0$ at $s=1\left(\beta_{*}=(\beta+1) /(\beta-1)\right.$ if $\beta>1$, $\beta_{*}=1$ if $\left.\beta=1\right)$. It follows easily from this that any positive solution of (4.2) is either identically one (if $E(u)=\beta_{*}$ ), or if $E(u)>\beta_{*}$, then $u$ is periodic with minimum value $a \in(0,1)$ and maximum value $b \in(1, \infty)$ determined by

$$
F(a)=F(b)=E(u) .
$$

The period $T$ of such a solution is given by

$$
T=2 \int_{a}^{b} \frac{d s}{\sqrt{F(a)-F(s)}},
$$

and by Lemma 5.8 ,

$$
\lim _{a \rightarrow 0^{+}} T=\pi, \quad \lim _{a \rightarrow 1^{-}} T=\frac{2 \pi}{\sqrt{1+\beta}} .
$$

By continuity of $T$ with respect to $a, T$ takes every value between these two limits. As in Theorem 1, these solutions give rise to closed locally convex curves precisely when the period is a rational multiple of $2 \pi$. In the case $\beta=1$ these are the curves of Mullins [8] and Abresch and Langer [1]. For $\beta>k^{2}-1$ and $T=2 \pi / k, k=3,4,5, \cdots$, we obtain the convex embedded curves of Andrews [2].

Case 2. $\beta \in(0,1)$. In this case we obtain periodic solutions similar to those above, and in addition, solutions similar to those described in Theorem 2 . If $\beta \in(0,1)$, then $F(s)=0$ at $s=0$ and at $s=\beta_{1}=[2 /(1-\beta)]^{1 /(1+\beta)}>1$, and $F$ is strictly convex with minimum value $\beta_{*}=(\beta+1) /(\beta-1)<0$ at $s=1$. Similarly to above we see that solutions with energy $E(u) \in\left(\beta_{*}, 0\right)$ are periodic with minimum and maximum values $a \in(0,1)$ and $b \in\left(1, \beta_{1}\right)$ determined by (4.5). The period is again given by (4.6), and by Lemma 5.9 ,

$$
\lim _{a \rightarrow 0^{+}} T=\frac{2 \pi}{1+\beta}, \quad \lim _{a \rightarrow 1^{-}} T=\frac{2 \pi}{\sqrt{1+\beta}} .
$$


For $E(u)=0$ we obtain the solutions with minimum value $a=0$ and maximum value $b=\beta_{1}$. These satisfy $u^{\prime}=0$ where $u=0$, so they extend to periodic functions with period $T=2 \pi /(1+\beta)$. The curves determined by these solutions are embedded convex curves of total curvature $k=2 \pi /(1+\beta)$, the two ends of which meet at the origin and $\kappa \rightarrow 0$ at the endpoints. If $2 \pi /(1+\beta)$ is a rational multiple of $2 \pi$, these curves can be continued periodically to obtain $C^{2}$ locally convex immersed curves similar to the Mullins-Abresch-Langer curves, except that the origin lies on the curve and is a point of self-intersection. Moreover, it is easy to see that in certain cases the origin is the only point of self-intersection. Namely, when $\beta=1 /(2 k-1)$ the curve is a " $2 k$-leaved clover" if $k \geq 2$ is even and a " $k$-leaved clover" if $k \geq 3$ is odd.

Finally, if $E(u)>0$, we obtain a translation of the solution of (4.2) with initial conditions $u(0)=a>\beta_{1}, u^{\prime}(0)=0$, so $u$ is defined on a bounded interval $[-T, T]$ with $u( \pm T)=0$ and $\left|u^{\prime}( \pm T)\right|^{2}=E(u)>0$. In addition,

$$
T=\int_{0}^{a} \frac{d s}{\sqrt{F(a)-F(s)}} .
$$

The curve $\gamma$ determined by such a solution is a finite embedded convex curve of finite total curvature $k$, with its endpoints on $\partial B_{r}(0)$ for $r=\sqrt{E(u)}=\sqrt{F(a)}$. From Lemma 5.10 we see that $T$ is strictly decreasing with respect to $a$ with

$$
\lim _{a \rightarrow \beta_{1}^{+}} k=\frac{2 \pi}{1+\beta}, \quad \lim _{a \rightarrow \infty} k=\pi .
$$

In addition, $\gamma$ is tangent to a line through the origin at each endpoint, and $\kappa \rightarrow 0$ at the endpoints. Thus by attaching a suitable semi-infinite portion of each of these lines to $\gamma$ we obtain a complete, properly immersed, locally convex curve of class $C^{2}$ (at least), which moves homothetically by $\kappa^{\alpha}$ flow. This procedure is of course also possible in the case $E(u)=0$. In addition, we may combine several such curves with a suitable number of portions of lines through the origin to obtain more elaborate examples.

Expanding Solutions. For $\lambda>0$ (4.1) has many complete noncompact embedded convex solutions. These were classified in [11] - they are either lines through the origin or embedded convex curves asymptotic to the boundary of a closed convex cone with vertex at the origin and vertex angle less than $\pi$ (the existence of such solutions in the case $\alpha=1$ has also been proved in [4,6]). It is clear that any convex solution of (4.1) with $\lambda>0$, whether complete or not, must be embedded and have total curvature less than $\pi$.

In [7] Ishimura proved the existence of expanding spiral-like solutions of (4.1) with $\lambda>0$ in the case $\alpha=1$. His solutions have one endpoint and spiral to infinity without self-intersection and with finite total curvature. Since any such solution can be extended to a complete solution which is symmetric about its point of maximum curvature, they are parts of embedded infinite solutions which are asymptotic to the boundary of a cone, as explained above.

To prove this we normalize so that $\lambda=1$ in (4.1). Then every convex solution of (4.1) corresponds to a negative solution of

$$
u^{\prime \prime}+u=(-u)^{-\beta} \text {. }
$$

But it is clear that any negative solution $u$ of (4.11) must satisfy $u^{\prime \prime} \geq C(\beta)>0$, which implies that any maximal negative solution exists only on some bounded interval and 
is zero at both endpoints. Thus after a translation we may assume that $u(0)=a<0$ and $u^{\prime}(0)=0$. It follows that $u$ is symmetric about its minimum. This implies that the curve determined by $u$ is symmetric about its point of maximal curvature.

Evidently there are no genuine spiral-like homothetic solutions of flow by positive powers of the curvature.

5. Some Lemmas. Here we prove some technical lemmas which were used in the previous sections. The first six of these concern the behaviour of certain integrals involving the function

$$
F(s)=s^{2}-\frac{2}{\beta+1} s^{\beta+1} .
$$

This function has its minimum $\beta_{*}=(\beta-1) /(\beta+1)<0$ at $s=1$ if $\beta \in(0,1)$, and it has its maximum $\beta_{*}=(\beta-1) /(\beta+1)>0$ at $s=1$ if $\beta>1$. It has two zeros, one at $s=0$ and the other at $s=\beta_{1}=[2 /(\beta+1)]^{1 /(1-\beta)}>1$.

LEMMA 5.1. Suppose that $\beta \in(0,1)$ and for each $a \in[0,1)$ let $b$ be the unique number in $\left(1, \beta_{1}\right]$ such that $F(b)=F(a)$. Let $T$ be defined by

$$
T(a)=\int_{a}^{b} \frac{d s}{\sqrt{F(a)-F(s)}} .
$$

Then

$$
\lim _{a \rightarrow 0^{+}} T(a)=\frac{\pi}{1-\beta}, \quad \lim _{a \rightarrow 1^{-}} T(a)=\frac{\pi}{\sqrt{1-\beta}}
$$

Proof. Since $F(a) \leq 0$ for $a \in\left[0, \beta_{1}\right]$, we have $F(a)-F(s) \leq-F(s)$. Thus by the monotone convergence theorem

$$
\begin{aligned}
\lim _{a \rightarrow 0} T(a) & \geq \lim _{a \rightarrow 0} \int_{a}^{b} \frac{d s}{\sqrt{-F(s)}} \\
& =\int_{0}^{\beta_{1}} \frac{d s}{\sqrt{-F(s)}} \\
& =\frac{\pi}{1-\beta} .
\end{aligned}
$$

The last integral can be evaluated by making the substitution

$$
t=\sqrt{(\beta+1) / 2} s^{(1-\beta) / 2} .
$$

Next we show the reverse inequality. Let $\epsilon>0$ be small, and suppose that $F(a)=F(b)=-\epsilon^{\gamma}$, where $\gamma>1$ is chosen so that $\beta \gamma<1$. Then $a \rightarrow 0$ and $b \rightarrow \beta_{1}$ as $\epsilon \rightarrow 0$, and

$$
\int_{a}^{b} \frac{d s}{\sqrt{F(a)-F(s)}}=\int_{\Sigma_{1}} \frac{d s}{\sqrt{F(a)-F(s)}}+\int_{\Sigma_{2}} \frac{d s}{\sqrt{F(a)-F(s)}}
$$

where

$$
\Sigma_{1}=\left\{s \in\left[0, \beta_{1}\right]: \epsilon^{\gamma} \leq-F(s) \leq \epsilon\right\}, \quad \Sigma_{2}=\left\{s \in\left[0, \beta_{1}\right]:-F(s)>\epsilon\right\} .
$$

To estimate the integral over $\Sigma_{1}$, first observe that for $\epsilon$ small $\Sigma_{1}$ is a union of two disjoint intervals $\left[a, a^{\prime}\right]$ and $\left[b^{\prime}, b\right]$ where $F\left(a^{\prime}\right)=F\left(b^{\prime}\right)=-\epsilon$. In addition, $F$ is concave 
on $\left[a, a^{\prime}\right]$ and convex on $\left[b^{\prime}, b\right]$. For $s \in\left[a, a^{\prime}\right]$ we have

$$
F(a)-F(s) \geq-F^{\prime}(a)(s-a)
$$

and hence

$$
\begin{aligned}
\int_{a}^{a^{\prime}} \frac{d s}{\sqrt{F(a)-F(s)}} & \leq \frac{1}{\sqrt{-F^{\prime}(a)}} \int_{a}^{a^{\prime}} \frac{d s}{\sqrt{s-a}} \\
& =\frac{2 \sqrt{a^{\prime}-a}}{\sqrt{-F^{\prime}(a)}} \\
& \leq C(\beta) \epsilon^{\delta}
\end{aligned}
$$

where $\delta=(1-\beta \gamma) /(2 \beta+2)>0$. Here we have used

$$
a \approx c(\beta) \epsilon^{\gamma /(\beta+1)}, \quad a^{\prime} \approx c(\beta) \epsilon^{1 /(\beta+1)}
$$

for a positive constant $c(\beta)$. Similarly, for $s \in\left[b^{\prime}, b\right]$

$$
F(b)-F(s) \geq F^{\prime}(s)(b-s) \geq F^{\prime}\left(b^{\prime}\right)(b-s),
$$

and hence

$$
\begin{aligned}
\int_{b^{\prime}}^{b} \frac{d s}{\sqrt{F(b)-F(s)}} & \leq \frac{1}{\sqrt{F^{\prime}\left(b^{\prime}\right)}} \int_{b^{\prime}}^{b} \frac{d s}{\sqrt{b-s}} \\
& =\frac{2 \sqrt{b-b^{\prime}}}{\sqrt{F^{\prime}\left(b^{\prime}\right)}} \\
& \leq C(\beta) \sqrt{\epsilon} .
\end{aligned}
$$

Here we have used the fact that near $s=\beta_{1}, F^{\prime}(s)$ is bounded between two positive constants depending only on $\beta$.

On $\Sigma_{2}$ we have $-F(s)>\epsilon=-\epsilon^{1-\gamma} F(a)$ and hence $F(a)-F(s) \geq-(1-$ $\left.\epsilon^{\gamma-1}\right) F(s)$, so

$$
\begin{aligned}
\int_{\Sigma_{2}} \frac{d s}{\sqrt{F(a)-F(s)}} & \leq \frac{1}{\sqrt{1-\epsilon^{\gamma-1}}} \int_{0}^{\beta_{1}} \frac{d s}{\sqrt{-F(s)}} \\
& \leq \frac{\pi}{(1-\beta) \sqrt{1-\epsilon^{\gamma-1}}}
\end{aligned}
$$

Combining the above estimates we obtain, since $0<\delta<1 / 2$,

$$
T(a) \leq \frac{\pi}{(1-\beta) \sqrt{1-\epsilon^{\gamma-1}}}+C \epsilon^{\delta}
$$

and hence

$$
\lim _{a \rightarrow 0} T(a) \leq \frac{\pi}{1-\beta}
$$

Next we prove the second equality in (5.3). It is convenient to split the integral in (5.2) into two integrals, the first over $[a, 1]$ and the second over $[1, b]$. Introducing the new variable $t$ defined by $s=(1-t) a+t$ we obtain

$$
\int_{a}^{1} \frac{d s}{\sqrt{F(a)-F(s)}}=\int_{0}^{1} \frac{(1-a) d t}{\sqrt{F(a)-F\left(a_{t}\right)}}
$$


where $a_{t}=(1-t) a+t$. We now show that

$$
\frac{(1-a)}{\sqrt{F(a)-F\left(a_{t}\right)}} \leq \frac{2}{\sqrt{F^{\prime \prime}(1) t}}
$$

for all $a \in(0,1]$ sufficiently close to 1 . By Taylor's theorem we have, for some $\hat{a}_{t} \in$ $\left[a, a_{t}\right]$

$$
\begin{aligned}
F\left(a_{t}\right) & =F(a)+F^{\prime}(a)\left(a_{t}-a\right)+\frac{1}{2} F^{\prime \prime}\left(\hat{a}_{t}\right)\left(a_{t}-a\right)^{2} \\
& \leq F(a)+F^{\prime}(a)\left(a_{t}-a\right)+\frac{1}{2} F^{\prime \prime}(1)\left(a_{t}-a\right)^{2}
\end{aligned}
$$

since $F^{\prime \prime \prime}>0$. In addition, for some $\hat{a} \in[a, 1]$ we have

$$
F^{\prime}(a)=F^{\prime}(1)+F^{\prime \prime}(\hat{a})(a-1) \leq F^{\prime \prime}(a)(a-1)
$$

since $F^{\prime}(1)=0$ and $F^{\prime \prime \prime}>0$. Thus

$$
\begin{aligned}
F(a)-F\left(a_{t}\right) & \geq F^{\prime \prime}(a)(1-a)^{2} t-\frac{1}{2} F^{\prime \prime}(1)(1-a)^{2} t^{2} \\
& \geq \frac{1}{4} F^{\prime \prime}(1)(1-a)^{2} t
\end{aligned}
$$

provided $a$ is so close to 1 that

$$
F^{\prime \prime}(a) \geq \frac{3}{4} F^{\prime \prime}(1)
$$

(5.9) now follows.

We now use Taylor's theorem again to write

$$
\begin{aligned}
F\left(a_{t}\right)= & F(a)+F^{\prime}(a)\left(a_{t}-a\right)+\frac{1}{2} F^{\prime \prime}(a)\left(a_{t}-a\right)^{2}+O\left(\left|a_{t}-a\right|^{3}\right) \\
= & F(a)+\frac{F^{\prime}(a)-F^{\prime}(1)}{1-a}(1-a)^{2} t+\frac{1}{2} F^{\prime \prime}(a)(1-a)^{2} t^{2} \\
& +O\left(|1-a|^{3} t^{3}\right) .
\end{aligned}
$$

Using the dominated convergence theorem, which is permissible by (5.9), we see that

$$
\begin{aligned}
\lim _{a \rightarrow 1} \int_{0}^{1} \frac{(1-a) d t}{\sqrt{F(a)-F\left(a_{t}\right)}} & =\int_{0}^{1} \frac{d t}{\sqrt{F^{\prime \prime}(1) t-F^{\prime \prime}(1) t^{2} / 2}} \\
& =\frac{\pi}{\sqrt{2 F^{\prime \prime}(1)}} \\
& =\frac{\pi}{2 \sqrt{1-\beta}} .
\end{aligned}
$$

Similarly,

$$
\lim _{a \rightarrow 1} \int_{1}^{b} \frac{d s}{\sqrt{F(a)-F(s)}}=\frac{\pi}{2 \sqrt{1-\beta}},
$$

and the second equality of (5.3) follows.

Remark. For the proof just given it sufficient that $F \in C^{2}$ with $F^{\prime \prime}(1)>0$. Only a few minor modifications need to be made where we have used the fact that $F^{\prime \prime \prime}>0$. 
LEMMA 5.2. Let $\beta \in(0,1)$, and for $a \in\left[\beta_{1}, \infty\right)$ define

$$
T(a)=\int_{0}^{a} \frac{d s}{\sqrt{F(a)-F^{\prime}(s)}} .
$$

Then $T$ is strictly decreasing,

$$
T\left(\beta_{1}\right)=\frac{\pi}{1-\beta}
$$

and

$$
\lim _{a \rightarrow \beta_{1}^{+}} T(a)=\frac{\pi}{1-\beta}, \quad \lim _{a \rightarrow \infty} T(a)=\frac{\pi}{2} .
$$

Proof. First observe that since $F$ is convex for $s \geq \beta_{1}$ and $F^{\prime}\left(\beta_{1}\right)$ is positive, $F(a)-F(s) \geq F^{\prime}\left(\beta_{1}\right)(a-s)$ for $s \in\left[\beta_{1}, a\right]$, and hence

$$
\begin{aligned}
\int_{\beta_{1}}^{a} \frac{d s}{\sqrt{F(a)-F(s)}} & \leq \frac{1}{\sqrt{F^{\prime}\left(\beta_{1}\right)}} \int_{\beta_{1}}^{a} \frac{d s}{\sqrt{a-s}} \\
& =2 \sqrt{\frac{a-\beta_{1}}{F^{\prime}\left(\beta_{1}\right)}} \\
& \rightarrow 0 \text { as } a \rightarrow \beta_{1}^{+} .
\end{aligned}
$$

Next, $F(a)$ decreases monotonically to 0 as $a \rightarrow \beta_{1}^{+}$, so by the monotone convergence theorem

$$
\begin{aligned}
\lim _{a \rightarrow \beta_{1}^{+}} T & =\lim _{a \rightarrow \beta_{1}^{+}} \int_{0}^{a} \frac{d s}{\sqrt{F(a)-F(s)}} \\
& =\lim _{a \rightarrow \beta_{1}^{+}}\left\{\int_{0}^{\beta_{1}} \frac{d s}{\sqrt{F(a)-F(s)}}+\int_{\beta_{1}}^{a} \frac{d s}{\sqrt{F(a)-F(s)}}\right\} \\
& =\int_{0}^{\beta_{1}} \frac{d s}{\sqrt{-F(s)}} \\
& =\frac{\pi}{1-\beta} .
\end{aligned}
$$

This proves the first equality in (5.12), and of course, also (5.11).

We now prove the second. For $0 \leq s \leq a$ we have

$$
F(a)-F(s)=a^{2}-s^{2}-\frac{2}{\beta+1}\left(a^{\beta+1}-s^{\beta+1}\right) \leq a^{2}-s^{2},
$$

and hence

$$
\int_{0}^{a} \frac{d s}{\sqrt{F(a)-F(s)}} \geq \int_{0}^{a} \frac{d s}{\sqrt{a^{2}-s^{2}}}=\frac{\pi}{2} .
$$

Now let $\epsilon>0$ be small. Then

$$
\begin{aligned}
\frac{2}{\beta+1}\left(a^{\beta+1}-s^{\beta+1}\right) & =\int_{s^{2}}^{a^{2}} t^{(\beta-1) / 2} d t \\
& \leq s^{(\beta-1) / 2}\left(a^{2}-s^{2}\right) \\
& \leq \epsilon\left(a^{2}-s^{2}\right)
\end{aligned}
$$


for $s$ sufficiently large, say $s \geq s_{0}=s_{0}(\beta, \epsilon)$. It follows that for $s \geq s_{0}$

$$
F(a)-F(s) \geq(1-\epsilon)\left(a^{2}-s^{2}\right)
$$

and hence

$$
\begin{aligned}
& \lim _{a \rightarrow \infty} \int_{0}^{a} \frac{d s}{\sqrt{F(a)-F(s)}} \\
= & \lim _{a \rightarrow \infty} \int_{0}^{s_{0}} \frac{d s}{\sqrt{F(a)-F(s)}}+\lim _{a \rightarrow \infty} \int_{s_{0}}^{a} \frac{d s}{\sqrt{F(a)-F(s)}} \\
\leq & \lim _{a \rightarrow \infty} \frac{1}{\sqrt{1-\epsilon}} \int_{s_{0}}^{a} \frac{d s}{\sqrt{a^{2}-s^{2}}} \\
= & \frac{\pi}{2 \sqrt{1-\epsilon}}
\end{aligned}
$$

by the dominated convergence theorem. The second equality of (5.12) now follows.

To prove that $T$ is strictly decreasing we introduce the new variable $t$ defined by $s=t a$. Then

$$
T=\int_{0}^{a} \frac{d s}{\sqrt{F(a)-F(s)}}=\int_{0}^{1} \frac{a d t}{\sqrt{F(a)-F(t a)}},
$$

and

$$
F(a)-F(t a)=a^{2}\left(1-t^{2}\right)-\frac{2}{\beta+1} a^{\beta+1}\left(1-t^{\beta+1}\right),
$$

so the result follows directly by differentiating the integrand with respect to $a$.

Lemma 5.3. Let $\beta>1$ and for $a \in(0,1)$ define $T$ by (5.10). Then $T$ is strictly increasing, $T(a)>\pi / 2$ and

$$
\lim _{a \rightarrow 0^{+}} T(a)=\frac{\pi}{2}, \quad \lim _{a \rightarrow 1^{-}} T(a)=\infty .
$$

Proof. For $0 \leq s \leq a(5.13)$ holds with strict inequality unless $s=a$. Thus

$$
\int_{0}^{a} \frac{d s}{\sqrt{F(a)-F(s)}}>\int_{0}^{a} \frac{d s}{\sqrt{a^{2}-s^{2}}}=\frac{\pi}{2} .
$$

Now let $\epsilon>0$ be small. Then for sufficiently small $a>0$ we have, since $\beta>1$,

$$
F(a)-F(s) \geq(1-\epsilon)\left(a^{2}-s^{2}\right) \text { for } 0 \leq s \leq a,
$$

and hence

$$
\int_{0}^{a} \frac{d s}{\sqrt{F(a)-F(s)}} \leq \frac{1}{\sqrt{1-\epsilon}} \int_{0}^{a} \frac{d s}{\sqrt{a^{2}-s^{2}}}=\frac{\pi}{2 \sqrt{1-\epsilon}}
$$

The first equality of (5.14) follows.

We now prove the second. First we note that since now $\beta>1, F^{\prime \prime \prime}<0$. Thus by Taylor's theorem, for $0 \leq s \leq a$ we have

$$
F(s) \geq F(a)+F^{\prime}(a)(s-a)+\frac{1}{2} F^{\prime \prime}(a)(s-a)^{2} .
$$

Since $F^{\prime}(1)=0$, for some $\hat{a} \in[a, 1]$ we also have

$$
F^{\prime}(a)=-F^{\prime \prime}(\hat{a})(1-a) \text {. }
$$


Thus for $0 \leq s \leq 2 a-1 \leq a$ we have

$$
\begin{aligned}
F(a)-F(s) & \leq F^{\prime}(a)(a-s)-\frac{1}{2} F^{\prime \prime}(a)(s-a)^{2} \\
& =-F^{\prime \prime}(\hat{a})(1-a)(a-s)-\frac{1}{2} F^{\prime \prime}(a)(s-a)^{2} \\
& \leq-\frac{3}{2} F^{\prime \prime}(1)(a-s)^{2}
\end{aligned}
$$

since $F^{\prime \prime}(1) \leq F^{\prime \prime}(\hat{a}) \leq F^{\prime \prime}(a)<0$ for $a$ close to 1 . It follows that

$$
\begin{aligned}
\int_{0}^{a} \frac{d s}{\sqrt{F(a)-F(s)}} & \geq \frac{\sqrt{2}}{\sqrt{-3 F^{\prime \prime}(1)}} \int_{0}^{2 a-1} \frac{d s}{a-s} \\
& =\frac{\sqrt{2}}{\sqrt{-3 F^{\prime \prime}(1)}}[\log a-\log (1-a)] \\
& \rightarrow \infty \text { as } a \rightarrow 1^{-}
\end{aligned}
$$

Finally, the strict monotonicity of $T$ can be proved as in Lemma 5.2.

LEMMA 5.4. Let $\beta>1$ and for $a \in(1, \infty)$ define

$$
T(a)=\int_{a}^{\infty} \frac{d s}{\sqrt{F(a)-F(s)}} .
$$

Then $T$ is strictly decreasing and

$$
\lim _{a \rightarrow 1^{+}} T(a)=\infty, \quad \lim _{a \rightarrow \infty} T(a)=0 .
$$

Proof. Since $F$ is concave for $s \geq 1$, we see that for $s \geq a>1$ we have

$$
F(a)-F(s) \geq-F^{\prime}(a)(s-a)=2\left(a^{\beta}-a\right)(s-a) .
$$

In addition, for large $s$ we have $F(a)-F(s)=O\left(s^{\beta+1}\right)$. Using these facts and the fact that $\beta>1$ we easily see that $T<\infty$ for any $a \in(1, \infty)$. To see that $T$ is strictly decreasing we rewrite $T$ as

$$
T=\int_{0}^{\infty} \frac{d s}{\sqrt{F(a)-F(a+s)}}
$$

By direct computation we see that the integrand in (5.17) is strictly decreasing with respect to $a$ because $F^{\prime \prime}(s)<0$ for $s>1$. The proof of the first equality in (5.16) is almost identical to that of the second equality in (5.14) and is therefore omitted. The second follows from the dominated convergence theorem since the integrand in (5.17) decreases pointwise to zero as $a \rightarrow \infty$.

LEMMA 5.5. Let $\beta>1$ and for $\eta>\beta_{*}$ define

$$
T(\eta)=\int_{0}^{\infty} \frac{d s}{\sqrt{\eta-F(s)}} .
$$

Then $T$ is strictly decreasing and

$$
\lim _{\eta \rightarrow \beta_{*}^{+}} T(\eta)=\infty, \quad \lim _{\eta \rightarrow \infty} T(\eta)=0 .
$$


Proof. $T$ is finite because $F(s) \leq \beta_{*}<\eta$ and $\eta-F(s)=O\left(s^{\beta+1}\right)$ for large $s$. It is obvious that $T$ is strictly decreasing. To prove the first equality of (5.19) we set $\eta=F(a)$ for $a>1$, so that $\eta \rightarrow \beta_{1}^{+}$as $a \rightarrow 1^{+}$. The result then follows from Lemma 5.4. The second equality of (5.19) follows with the aid of the dominated convergence theorem as above.

LEMMA 5.6. Let $\beta>1$ and for $a \in(1, \infty)$ define

$$
T(a)=\int_{a}^{\infty} \frac{d s}{\sqrt{\beta_{*}-F(s)}} .
$$

Then $T$ is finite for all $a>1$ and

$$
\lim _{a \rightarrow 1^{+}} T(a)=\infty
$$

The proof is similar to previous proofs so it is omitted.

The following lemma concerns the function

$$
F(s)=s^{2}+\frac{2}{\beta+1} s^{\beta+1},
$$

with $\beta>0, \beta \neq 1$.

Lemma 5.7. For any $a \in(0, \infty)$ let $T$ be defined by $(5.10)$.

(i) If $0<\beta<1$, then $T$ is strictly increasing with

$$
\lim _{a \rightarrow 0^{+}} T=0, \quad \lim _{a \rightarrow \infty} T=\frac{\pi}{2} .
$$

(ii) If $\beta>1$, then $T$ is strictly decreasing with

$$
\lim _{a \rightarrow 0^{+}} T=\frac{\pi}{2}, \quad \lim _{a \rightarrow \infty} T=0 .
$$

Proof. First suppose that $0<\beta<1$. For $0 \leq s \leq a$ we have

$$
F(a)-F(s) \geq \frac{2}{\beta+1}\left(a^{\beta+1}-s^{\beta+1}\right),
$$

so if we make the change of variable $r=s / a$, we find that

$$
\begin{aligned}
\int_{0}^{a} \frac{d s}{\sqrt{F(a)-F(s)}} & \leq C(\beta) a^{(1-\beta) / 2} \\
& \rightarrow 0 \text { as } a \rightarrow 0^{+}
\end{aligned}
$$

where

$$
C(\beta)=\sqrt{\frac{\beta+1}{2}} \int_{0}^{1} \frac{d r}{\sqrt{1-r^{\beta+1}}} \leq \sqrt{2(\beta+1)} .
$$

On the other hand, given any $\epsilon>0$, there is a number $s_{0}=s_{0}(\epsilon, \beta)$ such that

$$
F(a)-F(s) \leq(1+\epsilon)\left(a^{2}-s^{2}\right) \text { for } s_{0} \leq s \leq a,
$$


and hence

$$
\begin{aligned}
\int_{s_{0}}^{a} \frac{d s}{\sqrt{F(a)-F(s)}} & \geq \frac{1}{\sqrt{1+\epsilon}} \int_{s_{0}}^{c_{1}} \frac{d s}{\sqrt{a^{2}-s^{2}}} \\
& \rightarrow \frac{\pi}{2 \sqrt{1+\epsilon}} \text { as } a \rightarrow \infty
\end{aligned}
$$

Since also

$$
\lim _{a \rightarrow \infty} \int_{0}^{s_{0}} \frac{d s}{\sqrt{F(a)-F(s)}}=0
$$

by the monotone convergence theorem, the second equality of (5.23) follows. The strict monotonicity of $T$ can be proved as in Lemma, 5.2.

Finally, the proof in the case $\beta>1$ is very similar and is omitted.

In the following two lemmas we let

$$
F(s)= \begin{cases}s^{2}-\frac{2}{1-\beta} s^{1-\beta} & \text { if } \beta \neq 1, \\ s^{2}-2 \log s & \text { if } \quad \beta=1\end{cases}
$$

Then for $\beta \geq 1, F(s) \rightarrow \infty$ as $s \rightarrow 0^{+}$and $s \rightarrow \infty, F$ is strictly convex and achieves its minimum value $\beta_{*}=(\beta+1) /(\beta-1)$ if $\beta>1\left(\beta_{*}=1\right.$ if $\left.\beta=1\right)$ at $s=1$. If $\beta \in(0,1)$, then $F(s)=0$ at $s=0$ and at $s=\beta_{1}==[2 /(1-\beta)]^{1 /(1+\beta)}, F$ is strictly convex and achieves its minimum value $\beta_{*}=(\beta+1) /(\beta-1)$ at $s=1$.

LEMMA 5.8. Suppose that $\beta \geq 1$ and for each $a \in(0,1]$ let $b$ be the unique number in $[1, \infty)$ such that $F(b)=F(a)$. Let $T$ be defined by $(5.2)$. Then

$$
\lim _{a \rightarrow 0^{+}} T(a)=\frac{\pi}{2}, \quad \lim _{a \rightarrow 1^{-}} T(a)=\frac{\pi}{\sqrt{1+\beta}} .
$$

Proof. To prove the first equality of (5.26) we split the interval of integration into the two intervals $[a, 1]$ and $[1, b]$. For $s \in[a, 1]$ we have, by Taylor's theorem and the fact that $F^{\prime \prime \prime}<0$,

$$
F(a)-F(s) \geq-F^{\prime}(a)(s-a)-\frac{1}{2} F^{\prime \prime}(a)(s-a)^{2} .
$$

The right hand side is clearly nonnegative for $s \in\left[a, a_{0}\right]$ for some $a_{0}>a$. In fact, a computation shows that for $a$ sufficiently small we may take $a_{0}=a+a / \beta$. In addition, setting

$$
\mu=\frac{F^{\prime \prime}(a)}{-2 F^{\prime}(a)}>0
$$

we see that

$$
\mu a \rightarrow \beta / 2 \text { as } a \rightarrow 0^{+} .
$$

Using these facts we now compute

$$
\begin{aligned}
\int_{a}^{a_{0}} \frac{d s}{\sqrt{F(a)-F(s)}} & \leq \frac{1}{\sqrt{-F^{\prime}(a)}} \int_{a}^{a_{0}} \frac{d s}{\sqrt{(s-a)-\mu(s-a)^{2}}} \\
& =\frac{2 \sqrt{2}}{\sqrt{F^{\prime \prime}(a)}} \arcsin \sqrt{\mu a / \beta} \\
& \rightarrow 0 \text { as } a \rightarrow 0^{+}
\end{aligned}
$$


since $F^{\prime \prime}(a) \rightarrow \infty$ as $a \rightarrow 0^{+}$.

Next, for any $\epsilon>0$ we have

$$
\lim _{a \rightarrow 0^{+}} \int_{\epsilon}^{1} \frac{d s}{\sqrt{F(a)-F(s)}}=0
$$

by the dominated convergence theorem, and also, for $a_{0}<\epsilon$,

$$
\int_{a_{0}}^{\epsilon} \frac{d s}{\sqrt{F(a)-F(s)}} \leq C(\beta) a^{(\beta-1) / 2}\left(\epsilon-a_{0}\right) .
$$

It follows that

$$
\lim _{a \rightarrow 0^{+}} \int_{a}^{1} \frac{d s}{\sqrt{F(a)-F(s)}}=0 .
$$

Next we have

$$
F(b)-F(s) \leq b^{2}-s^{2} \quad \text { for } \quad 1 \leq s \leq b,
$$

and for any small $\epsilon>0$ there is an $s_{0}=s_{0}(\epsilon, \beta)$ such that

$$
F(b)-F(s) \geq(1-\epsilon)\left(b^{2}-s^{2}\right) \text { for } s_{0} \leq s \leq b .
$$

It follows that

$$
\int_{1}^{b} \frac{d s}{\sqrt{F(b)-F(s)}} \geq \int_{1}^{b} \frac{d s}{\sqrt{b^{2}-s^{2}}} \rightarrow \frac{\pi}{2} \quad \text { as } \quad b \rightarrow \infty,
$$

and

$$
\int_{s_{0}}^{b} \frac{d s}{\sqrt{F(b)-F(s)}} \leq \frac{1}{\sqrt{1-\epsilon}} \int_{s_{0}}^{b} \frac{d s}{\sqrt{b^{2}-s^{2}}} \leq \frac{\pi}{2 \sqrt{1-\epsilon}} .
$$

In addition we have

$$
\lim _{b \rightarrow \infty} \int_{1}^{s_{0}} \frac{d s}{\sqrt{F(b)-F(s)}}=0
$$

by the dominated convergence theorem, so

$$
\lim _{b \rightarrow \infty} \int_{1}^{b} \frac{d s}{\sqrt{F(b)-F(s)}}=\frac{\pi}{2} .
$$

The first equality of (5.26) follows from (5.27) and (5.28).

The proof of the second equality of (5.26) is very similar to that of the second equality in (5.3), so it is omitted. Only a few minor changes need to be made because now $F^{\prime \prime \prime}<0$ instead of $F^{\prime \prime \prime}>0$.

LEMMA 5.9. Let $\beta \in(0,1)$, and for each $a \in[0,1]$ let $b \in\left[1, \beta_{1}\right]$ be the unique number such that $F(a)=F(b)$. Let $T$ be defined by (5.2). Then

$$
T(0)=\frac{\pi}{1+\beta}
$$

and

$$
\lim _{a \rightarrow 0^{+}} T(a)=\frac{\pi}{1+\beta}, \quad \lim _{a \rightarrow 1^{-}} T(a)=\frac{\pi}{\sqrt{1+\beta}} .
$$


Lemma 5.10. Let $\beta \in(0,1)$, and for $a \in\left[\beta_{1}, \infty\right)$ define $T$ by (5.10). Then $T$ is strictly decreasing,

$$
T\left(\beta_{1}\right)=\frac{\pi}{1+\beta}
$$

and

$$
\lim _{a \rightarrow \beta_{1}^{+}} T(a)=\frac{\pi}{1+\beta}, \quad \lim _{a \rightarrow \infty} T(a)=\frac{\pi}{2}
$$

The proofs of Lemmas 5.9 and 5.10 are almost identical to those of Lemmas 5.1 and 5.2, so they are omitted.

Acknowledgement. This work was done at the University of Bonn where the author was supported by an Alexander von Humboldt Fellowship.

\section{REFERENCES}

[1] U. ABRESCH AND J. LANGER, The normalized curve shortening flow and homothetic solutions, J. Diff. Geom., 23 (1986), pp. 175-196.

[2] B. Andrews, Evolving convex curves, Calc. Var., 7 (19@8), pp. 315-371.

[3] B. Chow AND R. Gulliver, Aleksandrov reflection and nonlinear evolution equations I. The $n$-sphere and $n$-ball, Calc. Var., 4 (1996), pp. 249-264.

[4] K. ECKer AND G. HuISkEN, Mean curvature evolution of entire graphs, Ann. Math., 130 (1989), pp. $453-471$.

[5] G. HUISKEN, On the expansion of convex hypersurfaces by the inverse of symmetric curvature functions, (unpublished).

[6] N. Ishimura, Curvature evolution of plane curves with prescribed opening angle, Bull. Austral. Math. Soc., 52 (1995), pp. 287-296.

[7] N. Ishimura, Shape of spirals, Tôhoku Math. J., 50 (1998), pp. 197-202.

[8] W. W. Mullins, Two dimensional motion of idealized grain boundaries, J. Appl. Phys., 27 (1956), pp. 900-904.

[9] R. SACKSTEDER, On hypersurfaces with no nonnegative sectional curvatures, Amer. J. Math., 82 (1960), pp. 609-630.

[10] J. URBAs, An expansion of convex surfaces, J. Diff. Geom., 33 (1991), pp. 91-125 and 35 (1992), pp. 763-765.

[11] J. URBAS, Complete noncompact self-similar solutions of Gauss curvature flows I. Positive powers, Math. Ann., 311 (1998), pp. 251-274.

[12] J. URBAS, Complete noncompact self-similar solutions of Gauss curvature flows II. Negative powers, Advances in Diff. Eqns, 4 (1999), pp. 323-346.

[13] J. van Heijenoort, On locally convex manifolds, Comm. Pure Appl. Math., 5 (1952), pp. 223242. 